\title{
Robust Adaptive Synchronization of the Energy Resource System with Constraint
}

\author{
Duo Meng \\ School of Civil and Architectural Engineering, Liaoning University of Technology, Jinzhou 121001, China \\ Correspondence should be addressed to Duo Meng; mengduo2004@163.com
}

Received 18 May 2013; Accepted 5 June 2013

Academic Editor: Tao Li

Copyright (C) 2013 Duo Meng. This is an open access article distributed under the Creative Commons Attribution License, which permits unrestricted use, distribution, and reproduction in any medium, provided the original work is properly cited.

\begin{abstract}
Two different chaos synchronization methods are proposed for a class of energy resource demand supply-system with input constraint. Firstly, chaotic synchronization is achieved for a class of energy resource demand supply system with known system parameters based on the Lyapunov theory. Secondly, an adaptive control approach is investigated for a class of energy resource demand supply system with input constraint, and the parameters of the system are unknown based on the Lyapunov stability and robust adaptive theory. To address the input constraint, new auxiliary signals and design systems are employed. Numerical simulations are provided to illustrate the effectiveness of the proposed approach.
\end{abstract}

\section{Introduction}

Energy resource system is a kind of complex nonlinear system. Energy resource including coal, petroleum, natural gas, water and electricity, and nuclear power can be classified as renewable energy and nonrenewable energy according to the capability of sustainable utilization. The issue of energy supply and demand has been valued worldwide with increasing development of economy. One of the most noticeable problems in the field of energy resource is how to study energy resource system deeply through nonlinear dynamics, which is currently a rapid developing method [1].

Reference [2] established a three-dimensional energy resource demand-supply system based on the real energy resources demand-supply in the East and the West of China. Furthermore, by adding a new variable to consider the renewable resources, a four-dimensional energy resource system was proposed in [3]. The dynamics behaviors of the fourdimensional energy resource system have been analyzed by means of the Lyapunov exponents and bifurcation diagrams. Also the same as the above-mentioned power systems, this four-dimensional energy resource system is with rich chaos behaviors. The problem of chaotic control for the energy resource system was considered in [1]. Feedback control and adaptive control methods were used to suppress chaos to unstable equilibrium or unstable periodic orbits, where only three of the system's parameters were supposed to be unknown. Reference [4] investigated the robust chaos synchronization problem for the four-dimensional energy resource systems based on the sliding mode control technique. The control of energy resource chaotic system was investigated by time delayed feedback control method in [5]. Based on stability criterion of linear system and Lyapunov stability theory, respectively, the chaos synchronization problems for energy resource demand-supply system were discussed using two novel different control methods in [6].

Although the adaptive synchronization control has achieved a great progress, the aforementioned control approaches assume that all the components of the considered energy resource demand-supply systems are in good operating conditions. As we know, many control systems have constraints on their inputs in the forms of input saturation or dead zone [7-17]. In practice, input saturation constraint is one of the most important input constraints which usually appear in many industries control systems. There are two main motivations for the saturation studies. One is that saturation is a potential problem for actuators of control systems. It often severely degrades the system performance, gives rise to undesirable inaccuracy, or even affects system stability. The other is that the control actions are usually limited in energy or magnitude; the saturation of the control output is necessary in practice. 


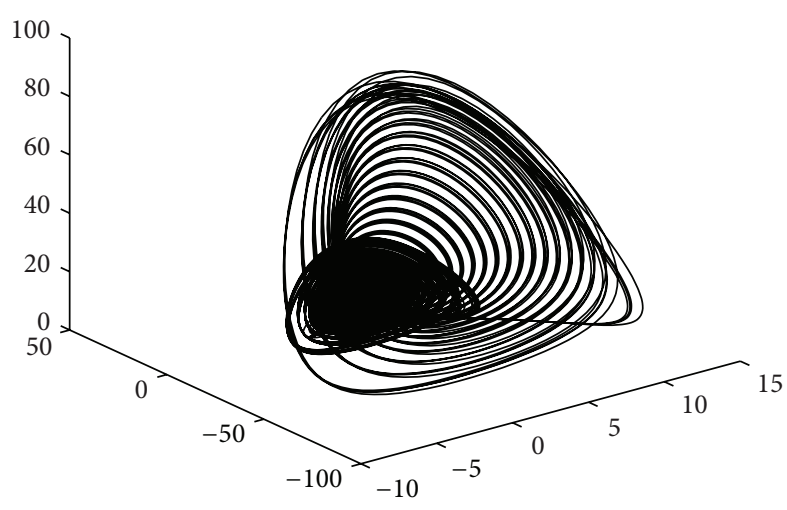

FIGURE 1: Energy resource attractor.

Motivated by the above observations, two different chaos synchronization control methods are proposed for a class of energy resource demand-supply system with input constraint. Based on Lyapunov stability and robust adaptive theory, on the assumption that all the parameters of the system are known and unknown, nonadaptive and adaptive control approaches are proposed to make the states of two chaotic systems asymptotic synchronization. The main contributions of the proposed algorithm are that (i) the problems of the input constraint are solved by employing a new auxiliary system; (ii) the stability of the energy resource demandsupply system is guaranteed based on the Lyapunov theory.

\section{Energy Resource Chaotic System}

In the paper, we consider the following energy resource system (see $[2,6])$ :

$$
\begin{gathered}
\dot{x}=a_{1} x\left(1-\frac{x}{M}\right)-a_{2}(y+z), \\
\dot{y}=-b_{1} y-b_{2} z+b_{3} x[N-(x-z)], \\
\dot{z}=c_{1} z\left(c_{2} x-c_{3}\right),
\end{gathered}
$$

where $x(t)$ is the energy resource shortage in A region, $y(t)$ is the energy resource supply increment in B region, and $z(t)$ is the energy resource import in A region; $M, N, a_{i}, b_{j}(i=1,2$, $j=1,2,3$ ) are parameters that are all positive real.

Similar to [6], when the system parameters are taken as the following values, this system exhibits chaotic behavior: $M=1.8, N=1, a_{1}=0.09, a_{2}=0.15, b_{1}=0.06, b_{2}=0.082$, $b_{3}=0.07, c_{1}=0.2, c_{2}=0.5$, and $c_{3}=0.4$. Without the particular statement, these values are adopted in this whole paper. Figure 1 shows the phase portrait with initial conditions of $x(0)=0.82, y(0)=0.29$, and $z(0)=0.48$.

\section{Synchronization of the Energy Resource System}

In this section, we will design a controller in order to make the response system trace the drive system. In order to obtain synchronization of the energy resource system (1), the drive system with subscript 1 is written as

$$
\begin{gathered}
\dot{x}_{1}=a_{1} x_{1}\left(1-\frac{x_{1}}{M}\right)-a_{2}\left(y_{1}+z_{1}\right), \\
\dot{y}_{1}=-b_{1} y_{1}-b_{2} z_{1}+b_{3} x_{1}\left[N-\left(x_{1}-z_{1}\right)\right], \\
\dot{z}_{1}=c_{1} z_{1}\left(c_{2} x_{1}-c_{3}\right) .
\end{gathered}
$$

The controlled response system with subscript 2 can be expressed as

$$
\begin{gathered}
\dot{x}_{2}=a_{1} x_{2}\left(1-\frac{x_{2}}{M}\right)-a_{2}\left(y_{2}+z_{2}\right)+u_{1}\left(v_{1}(t)\right), \\
\dot{y}_{2}=-b_{1} y_{2}-b_{2} z_{2}+b_{3} x_{2}\left[N-\left(x_{2}-z_{2}\right)\right]+u_{2}\left(v_{2}(t)\right), \\
\dot{z}_{2}=c_{1} z_{2}\left(c_{2} x_{2}-c_{3}\right)+u_{3}\left(v_{3}(t)\right),
\end{gathered}
$$

where $v_{i}(i=1,2,3)$ is the controller inputs to be designed, $u_{i}\left(v_{i}(t)\right)(i=1,2,3)$ denotes the plant input subject to saturation type nonlinearly.

Remark 1. If no input saturation (i.e., $\left.u_{i}\left(v_{i}(t)\right)=v_{i}(t)\right)(i=$ $1,2,3)$ is included in (3), then (3) becomes the chaotic systems studied widely; see $[2,6]$.

$u(v(t))$ is described by

$$
\begin{aligned}
u_{i}\left(v_{i}(t)\right) & =\operatorname{sat}\left(v_{i}(t)\right) \\
& = \begin{cases}\operatorname{sign}\left(v_{i}(t)\right) u_{M}, & \left|v_{i}(t)\right| \geq u_{i M}, \\
v_{i}(t), & \left|v_{i}(t)\right|<u_{i M},\end{cases}
\end{aligned}
$$

where $u_{i M}$ is a known bound of $u_{i}\left(v_{i}(t)\right)$. Clearly, the relationship between the applied control $u_{i}\left(v_{i}(t)\right)$ and the control input $v_{i}(t)$ has a sharp corner when $\left|v_{i}(t)\right|=u_{i M}$. Similar to [15], define

$$
\begin{aligned}
& e_{1}=x_{2}-x_{1}-h_{1}, \\
& e_{2}=y_{2}-y_{1}-h_{2}, \\
& e_{3}=z_{2}-z_{1}-h_{3},
\end{aligned}
$$

where $h_{i}(i=1,2,3)$ are filter signals and will be given later.

By using (2), (3), and (5), the error dynamical system can be written as

$$
\begin{aligned}
\dot{e}_{1}= & -\dot{h}_{1}+a_{1} e_{1}-a_{2}\left(e_{2}+e_{3}\right)-\frac{a_{1} x_{2}^{2}}{M}+\frac{a_{1} x_{1}^{2}}{M} \\
& +a_{1} h_{1}+a_{2}\left(h_{2}+h_{3}\right)+u_{1}\left(v_{1}(t)\right), \\
\dot{e}_{2}= & -\dot{h}_{2}-b_{1} e_{2}-b_{2} e_{3}+b_{3} N e_{1}-b_{3} x_{2}^{2} \\
& +b_{3} x_{1}^{2}+b_{3} x_{2} z_{2}-b_{3} x_{1} z_{1}+b_{1} h_{2} \\
& +b_{2} h_{3}-b_{3} N h_{1}+u_{2}\left(v_{2}(t)\right), \\
\dot{e}_{3}= & -\dot{h}_{3}-c_{1} c_{3} e_{3}+c_{1} c_{2} x_{2} z_{2} \\
& -c_{1} c_{2} x_{1} z_{1}+c_{1} c_{3} h_{3}+u_{3}\left(v_{3}(t)\right) .
\end{aligned}
$$


Similar to $[18,19]$, choose Lyapunov function candidate $V$ as

$$
V=\frac{1}{2} e_{1}^{2}+\frac{1}{2} e_{2}^{2}+\frac{1}{2} e_{3}^{2}
$$

The time derivative of $V$ is

$$
\dot{V}=e_{1} \dot{e}_{1}+e_{2} \dot{e}_{2}+e_{3} \dot{e}_{3} .
$$

Define the dynamic system as

$$
\dot{h}_{i}=-h_{i}+\left(u_{i}-v_{i}\right), \quad i=1,2,3 .
$$

Substituting (6) and (9) into (8) results in

$$
\begin{gathered}
\dot{V}=e_{1}\left[h_{1}+v_{1}+a_{1} e_{1}-a_{2}\left(e_{2}+e_{3}\right)-\frac{a_{1} x_{2}^{2}}{M}\right. \\
\left.+\frac{a_{1} x_{1}^{2}}{M}+a_{1} h_{1}+a_{2}\left(h_{2}+h_{3}\right)\right] \\
+e_{2}\left[h_{2}+v_{2}-b_{1} e_{2}-b_{2} e_{3}+b_{3} N e_{1}\right. \\
-b_{3} x_{2}^{2}+b_{3} x_{1}^{2}+b_{3} x_{2} z_{2}-b_{3} x_{1} z_{1} \\
\left.+b_{1} h_{2}+b_{2} h_{3}-b_{3} N h_{1}+u_{2}\right] \\
+e_{3}\left[h_{3}+v_{3}-c_{1} c_{3} e_{3}+c_{1} c_{2} x_{2} z_{2}\right. \\
\left.-c_{1} c_{2} x_{1} z_{1}+c_{1} c_{3} h_{3}\right] .
\end{gathered}
$$

By using Young's inequality, we have

$$
\begin{gathered}
-a_{2} e_{1}\left(e_{2}+e_{3}\right) \leq a_{2}^{2} e_{1}^{2}+\frac{1}{2} e_{2}^{2}+\frac{1}{2} e_{3}^{2}, \\
e_{2}\left[-b_{2} e_{3}+b_{3} N e_{1}\right] \leq \frac{b_{2}^{2}+b_{3}^{2} N^{2}}{2} e_{2}^{2}+\frac{1}{2} e_{3}^{2}+\frac{1}{2} e_{1}^{2} .
\end{gathered}
$$

Substituting (11) into (10) results in

$$
\begin{aligned}
\dot{V} \leq & \left(a_{1}+a_{2}^{2}+\frac{1}{2}\right) e_{1}^{2}+\left(b_{1}+\frac{1}{2}+\frac{b_{2}^{2}+b_{3}^{2} N^{2}}{2}\right) e_{2}^{2} \\
& +\left(1-c_{1} c_{3}\right) e_{3}^{2} \\
& +e_{1}\left[h_{1}+v_{1}-\frac{a_{1} x_{2}^{2}}{M}+\frac{a_{1} x_{1}^{2}}{M}+a_{1} h_{1}+a_{2}\left(h_{2}+h_{3}\right)\right] \\
& +e_{2}\left[h_{2}+v_{2}-b_{3} x_{2}^{2}+b_{3} x_{1}^{2}+b_{3} x_{2} z_{2}-b_{3} x_{1} z_{1}\right. \\
& \left.\quad+b_{1} h_{2}+b_{2} h_{3}-b_{3} N h_{1}\right] \\
& +e_{3}\left[h_{3}+v_{3}+c_{1} c_{2} x_{2} z_{2}-c_{1} c_{2} x_{1} z_{1}+c_{1} c_{3} h_{3}\right] .
\end{aligned}
$$

Choose the actual controllers $v_{i}$

$$
\begin{gathered}
v_{1}=-l_{1} e_{1}-h_{1}+\frac{a_{1} x_{2}^{2}}{M}-\frac{a_{1} x_{1}^{2}}{M}-a_{1} h_{1}-a_{2}\left(h_{2}+h_{3}\right), \\
v_{2}=-l_{2} e_{2}-h_{2}+b_{3} x_{2}^{2}-b_{3} x_{1}^{2}-b_{3} x_{2} z_{2} \\
\quad+b_{3} x_{1} z_{1}-b_{1} h_{2}-b_{2} h_{3}+b_{3} N h_{1} \\
v_{3}=-l_{3} e_{3}-h_{3}-c_{1} c_{2} x_{2} z_{2}+c_{1} c_{2} x_{1} z_{1}-c_{1} c_{3} h_{3},
\end{gathered}
$$

where $l_{i}(i=1,2,3)$ are positive design parameters. Substituting (13) into (12) results in

$$
\begin{aligned}
\dot{V} \leq & -\left(l_{1}-a_{1}-a_{2}^{2}-\frac{1}{2}\right) e_{1}^{2} \\
& -\left(l_{2}-b_{1}-\frac{1}{2}-\frac{b_{2}^{2}+b_{3}^{2} N^{2}}{2}\right) e_{2}^{2}-\left(l_{3}+c_{1} c_{3}-1\right) e_{3}^{2}
\end{aligned}
$$

Let $l_{i}$ satisfy $l_{1}>a_{1}+a_{2}^{2}+1 / 2, l_{2}>b_{1}+1 / 2+\left(b_{2}^{2}+b_{3}^{2} N^{2}\right) / 2$, and $l_{3}+c_{1} c_{3}>1$. We can obtain $\dot{V} \leq 0$, and it is concluded that $e_{1}, e_{2}$, and $e_{3}$ converge to zero as time $t$ tends to infinity. Therefore, the synchronization of response systems (3) and the drive system (2) is finally achieved.

Remark 2. It is noted that the controller in [6] was designed by using the stability criterion of linear system, not based on Lyapunov stability theory. However, this paper designed the control laws based on Lyapunov method. This paper can use a constructive way to obtain the control laws for this class of energy resource demand-supply system. In addition, the control laws' design parameters of this paper have fewer restrictions compared to [6].

\section{Simulation Results 1}

In this section, the initial values of are chosen as $x_{1}(0)=0.82$, $y_{1}(0)=0.29, z_{1}(0)=0.48, x_{2}(0)=0.69, y_{2}(0)=-0.03$, $z_{2}(0)=1.25$. The saturation values are $u_{1 M}=0.5, u_{2 M}=2$, and $u_{3 M}=2$. The design parameters in controllers are chosen as $l_{1}=20, l_{2}=10$, and $l_{3}=10$. The simulation results are shown in Figures 2, 3, 4, and 5.

\section{Adaptive Synchronization of the Energy Resource System}

In this section, we assume that all the parameters of the energy resource system are unknown. For convenience, we define $a_{3}=a_{1} / M, b_{4}=b_{3} N, d_{1}=c_{1} c_{2}$, and $d_{2}=c_{1} c_{3}$. The energy resource system (1) can be rewritten as:

$$
\begin{gathered}
\dot{x}=a_{1} x-a_{2}(y+z)-a_{3} x^{2}, \\
\dot{y}=-b_{1} y-b_{2} z-b_{3} x(x-z)+b_{4} x, \\
\dot{z}=d_{1} x z-d_{2} z
\end{gathered}
$$

and the drive system can be also rewritten as

$$
\begin{gathered}
\dot{x}_{1}=a_{1} x_{1}-a_{2}\left(y_{1}+z_{1}\right)-a_{3} x_{1}^{2}, \\
\dot{y}_{1}=-b_{1} y_{1}-b_{2} z_{1}-b_{3} x_{1}\left(x_{1}-z_{1}\right)+b_{4} x_{1}, \\
\dot{z}_{1}=d_{1} x_{1} z_{1}-d_{2} z_{1} .
\end{gathered}
$$



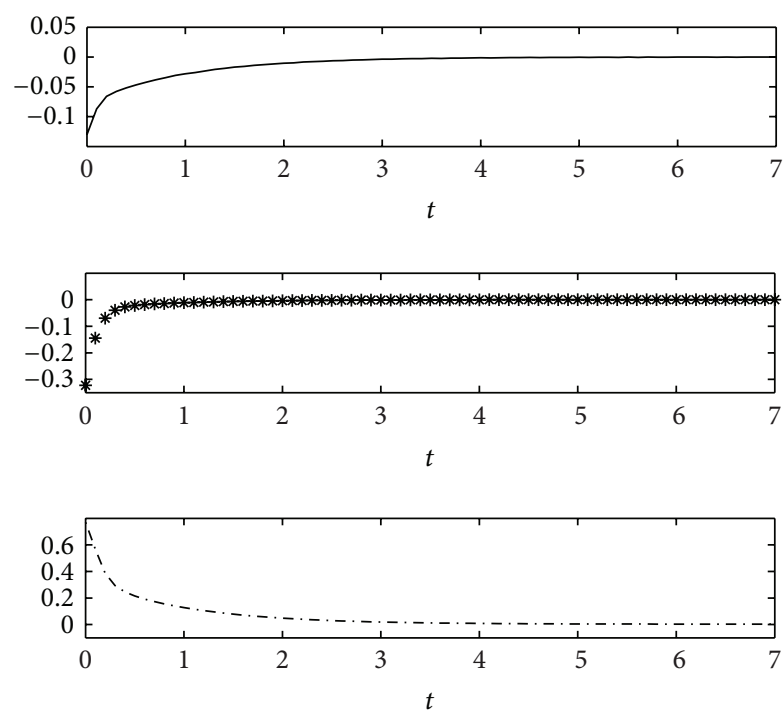

Figure 2: The trajectories of $e_{1}$ (solid line), $e_{2}$ (star line), and $e_{3}$ (dash-dotted line).

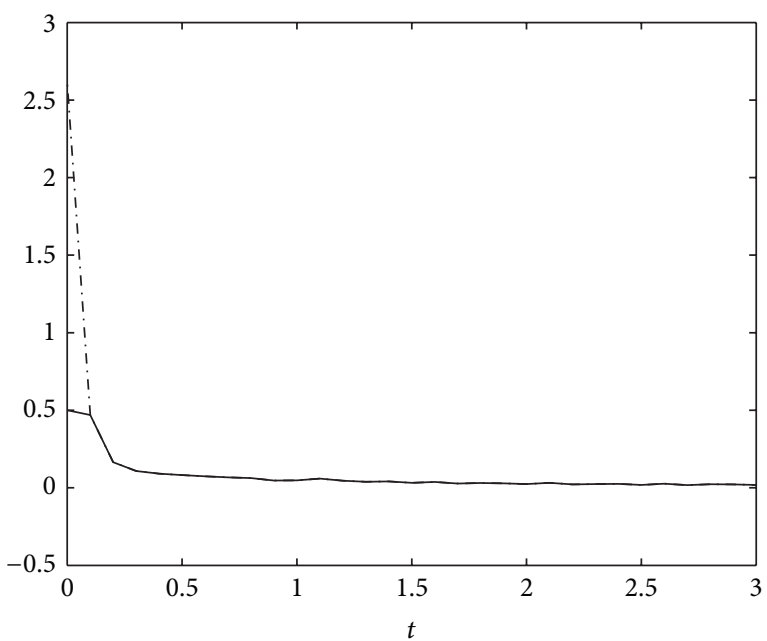

FIGURE 3: The trajectories of $v_{1}$ (solid line) and $u_{1}$ (dash-dotted line).

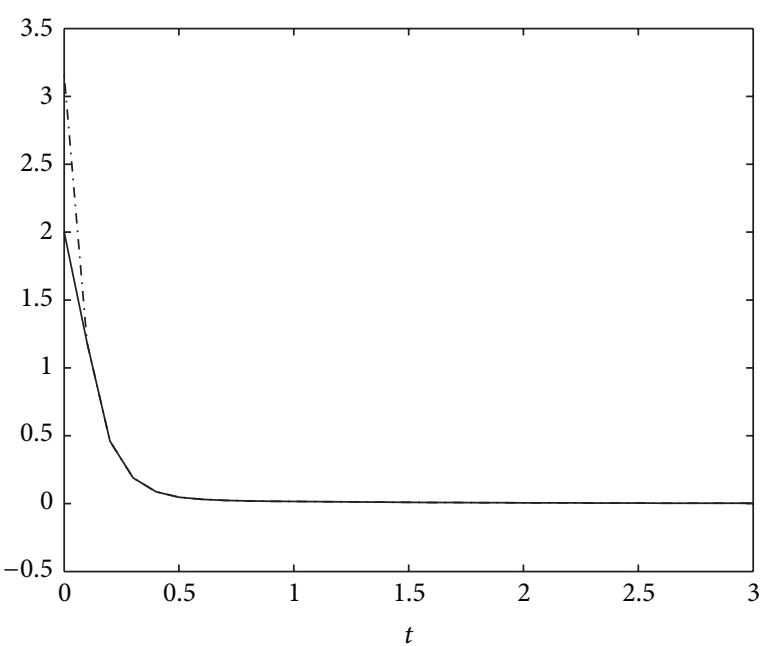

FIGURE 4: The trajectories of $v_{2}$ (solid line) and $u_{2}$ (dash-dotted line).

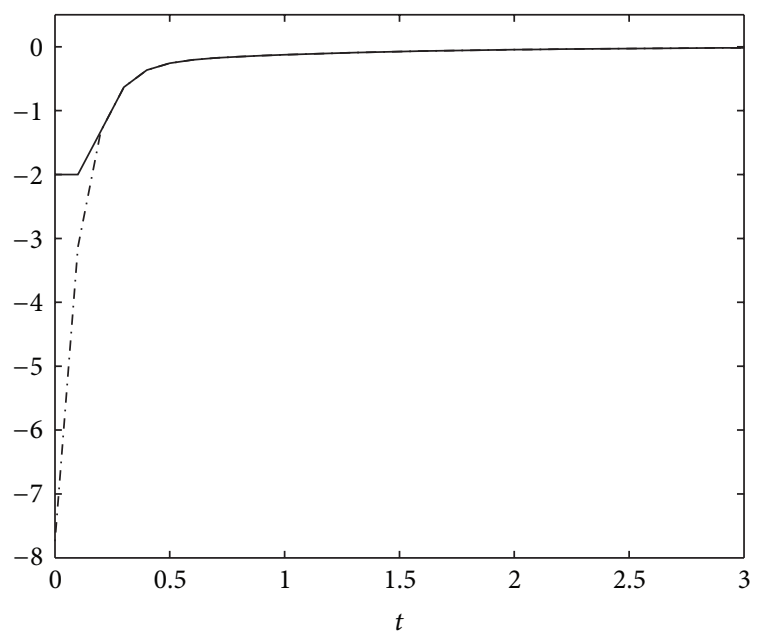

FIGURE 5: The trajectories of $v_{3}$ (solid line) and $u_{3}$ (dash-dotted line).

The response system can be expressed as

$$
\begin{gathered}
\dot{x}_{2}=a_{1} x_{2}-a_{2}\left(y_{2}+z_{2}\right)-a_{3} x_{2}^{2}+u_{1}\left(v_{1}(t)\right), \\
\dot{y}_{2}=-b_{1} y_{2}-b_{2} z_{2}-b_{3} x_{2}\left(x_{2}-z_{2}\right)+b_{4} x_{2}+u_{2}\left(v_{2}(t)\right), \\
\dot{z}_{2}=d_{1} x_{2} z_{2}-d_{2} z_{2}+u_{3}\left(v_{3}(t)\right) .
\end{gathered}
$$

Choose the same errors $e_{i}$ as (5) and the same filter signals $h_{i}$ as (9), and we have

$$
\begin{gathered}
\dot{e}_{1}=-\dot{h}_{1}+a_{1} e_{1}-a_{2}\left(e_{2}+e_{3}\right)-a_{3} x_{2}^{2}+a_{3} x_{1}^{2} \\
+a_{1} h_{1}+a_{2}\left(h_{2}+h_{3}\right)+u_{1}\left(v_{1}(t)\right), \\
\dot{e}_{2}=-\dot{h}_{2}-b_{1} e_{2}-b_{2} e_{3}+b_{4} e_{1}-b_{3} x_{2}^{2}+b_{3} x_{1}^{2}+b_{3} x_{2} z_{2} \\
-b_{3} x_{1} z_{1}+b_{1} h_{2}+b_{2} h_{3}-b_{4} h_{1}+u_{2}\left(v_{2}(t)\right), \\
\dot{e}_{3}=-\dot{h}_{3}-d_{2} e_{3}+d_{1} x_{2} z_{2}-d_{2} x_{1} z_{1}+d_{1} h_{3}+u_{3}\left(v_{3}(t)\right) .
\end{gathered}
$$

Choose the Lyapunov function candidate $\bar{V}$ as

$$
\begin{gathered}
\bar{V}=\frac{1}{2}\left(e_{1}^{2}+e_{2}^{2}+e_{3}^{2}+\widetilde{a}_{1}^{2}+\widetilde{a}_{2}^{2}+\widetilde{a}_{3}^{2}+\widetilde{b}_{1}^{2}+\widetilde{b}_{2}^{2}\right. \\
\left.+\widetilde{b}_{3}^{2}+\widetilde{b}_{4}^{2}+\widetilde{d}_{1}^{2}+\widetilde{d}_{2}^{2}\right),
\end{gathered}
$$

where $\tilde{a}_{i}=a_{i}-\widehat{a}_{i}(i=1,2,3), \tilde{b}_{j}=b_{j}-\widehat{b}_{j}(j=1,2,3,4)$, and $\tilde{d}_{k}=d_{k}-\widehat{d}_{k}(k=1,2)$.

The time derivative of $\bar{V}$ along with the solution of (18) is

$$
\begin{aligned}
\dot{\bar{V}}= & e_{1}\left[h_{1}+v_{1}+\widehat{a}_{1} e_{1}-\widehat{a}_{2}\left(e_{2}+e_{3}\right)-\widehat{a}_{3} x_{2}^{2}\right. \\
& \left.+\widehat{a}_{3} x_{1}^{2}+\widehat{a}_{1} h_{1}+\widehat{a}_{2}\left(h_{2}+h_{3}\right)\right] \\
+ & e_{2}\left[h_{2}+v_{2}-\widehat{b}_{1} e_{2}-\widehat{b}_{2} e_{3}+\widehat{b}_{4} e_{1}-\widehat{b}_{3} x_{2}^{2}\right. \\
& \left.+\widehat{b}_{3} x_{1}^{2}+\widehat{b}_{3} x_{2} z_{2}-\widehat{b}_{3} x_{1} z_{1}+\widehat{b}_{1} h_{2}+\widehat{b}_{2} h_{3}-\widehat{b}_{4} h_{1}\right] \\
+ & e_{3}\left[h_{3}+v_{3}-\widehat{d}_{2} e_{3}+\widehat{d}_{1} x_{2} z_{2}-\widehat{d}_{2} x_{1} z_{1}+\widehat{d}_{1} h_{3}\right]
\end{aligned}
$$




$$
\begin{aligned}
& +\tilde{a}_{1}\left(e_{1} h_{1}+e_{1}^{2}-\dot{\hat{a}}_{1}\right) \\
& +\widetilde{a}_{2}\left(-\left(e_{2}+e_{3}\right) e_{1}+\left(h_{2}+h_{3}\right) e_{1}-\dot{\hat{a}}_{2}\right) \\
& +\widetilde{a}_{3}\left(-e_{1} x_{2}^{2}+e_{1} x_{1}^{2}+e_{1} h_{1}+e_{1}\left(h_{2}+h_{3}\right)-\dot{\hat{a}}_{3}\right) \\
& +\tilde{b}_{1}\left(-e_{2}^{2}+e_{2} h_{2}-\dot{\hat{b}}_{1}\right)+\tilde{b}_{2}\left(-e_{2} e_{3}+e_{2} h_{3}-\dot{\hat{b}}_{2}\right) \\
& +\widetilde{b}_{3}\left(-e_{2} x_{2}^{2}+e_{2} x_{1}^{2}+e_{2} x_{2} z_{2}-e_{2} x_{1} z_{1}-\dot{\hat{b}}_{3}\right) \\
& +\tilde{b}_{4}\left(e_{1} e_{2}-e_{2} h_{1}-\dot{\hat{b}}_{4}\right) \\
& +\tilde{d}_{1}\left(e_{3} x_{2} z_{2}+e_{3} h_{3}-\dot{\hat{d}}_{1}\right)+\tilde{d}_{2}\left(-e_{3}^{2}-e_{3} x_{1} z_{1}-\dot{\hat{d}}_{2}\right) .
\end{aligned}
$$

Choose the actual controllers $v_{i}$ and update the laws of $\widehat{a}_{i}(i=$ $1,2,3), \widehat{b}_{j}(j=1,2,3,4)$, and $\widehat{d}_{k}(k=1,2)$ as follows:

$$
\begin{gathered}
v_{1}=-\bar{l}_{1} e_{1}-h_{1}-\widehat{a}_{1} e_{1}+\widehat{a}_{2}\left(e_{2}+e_{3}\right) \\
+\widehat{a}_{3} x_{2}^{2}-\widehat{a}_{3} x_{1}^{2}-\widehat{a}_{1} h_{1}-\widehat{a}_{2}\left(h_{2}+h_{3}\right), \\
v_{2}=-\bar{l}_{2} e_{2}-h_{2}+\widehat{b}_{1} e_{2}+\widehat{b}_{2} e_{3}-\widehat{b}_{4} e_{1}+\widehat{b}_{3} x_{2}^{2}-\widehat{b}_{3} x_{1}^{2} \\
-\widehat{b}_{3} x_{2} z_{2}+\widehat{b}_{3} x_{1} z_{1}-\widehat{b}_{1} h_{2}-\widehat{b}_{2} h_{3}+\widehat{b}_{4} h_{1}, \\
v_{3}=-\bar{l}_{3} e_{3}-h_{3}+\widehat{d}_{2} e_{3}-\widehat{d}_{1} x_{2} z_{2}+\widehat{d}_{2} x_{1} z_{1}-\widehat{d}_{1} h_{3},
\end{gathered}
$$

where $\bar{l}_{i}(i=1,2,3)$ are positive design parameters:

$$
\begin{gathered}
\dot{\hat{a}}_{1}=e_{1} h_{1}+e_{1}^{2}, \\
\dot{\hat{a}}_{2}=-\left(e_{2}+e_{3}\right) e_{1}+\left(h_{2}+h_{3}\right) e_{1}, \\
\dot{\hat{a}}_{3}=-e_{1} x_{2}^{2}+e_{1} x_{1}^{2}+e_{1} h_{1}+e_{1}\left(h_{2}+h_{3}\right), \\
\dot{\hat{b}}_{1}=-e_{2}^{2}+e_{2} h_{2}, \\
\dot{\hat{b}}_{2}=-e_{2} e_{3}+e_{2} h_{3}, \\
\dot{\hat{b}}_{3}=-e_{2} x_{2}^{2}+e_{2} x_{1}^{2}+e_{2} x_{2} z_{2}-e_{2} x_{1} z_{1}, \\
\dot{\hat{b}}_{4}=e_{1} e_{2}-e_{2} h_{1}, \\
\dot{\hat{d}}_{1}=e_{3} x_{2} z_{2}+e_{3} h_{3}, \\
\dot{\hat{d}}_{2}=-e_{3}^{2}-e_{3} x_{1} z_{1} .
\end{gathered}
$$

Substituting (21) and (22) into (20) results in

$$
\dot{\bar{V}}=-\bar{l}_{1} e_{1}^{2}-\bar{l}_{2} e_{2}^{2}-\bar{l}_{3} e_{3}^{2}
$$

From (23), we can conclude that the states $x_{2}, y_{2}$, and $z_{2}$ of response system (17) and the states $x_{1}, y_{1}$, and $z_{1}$ of drive system (16) are ultimately synchronized asymptotically.
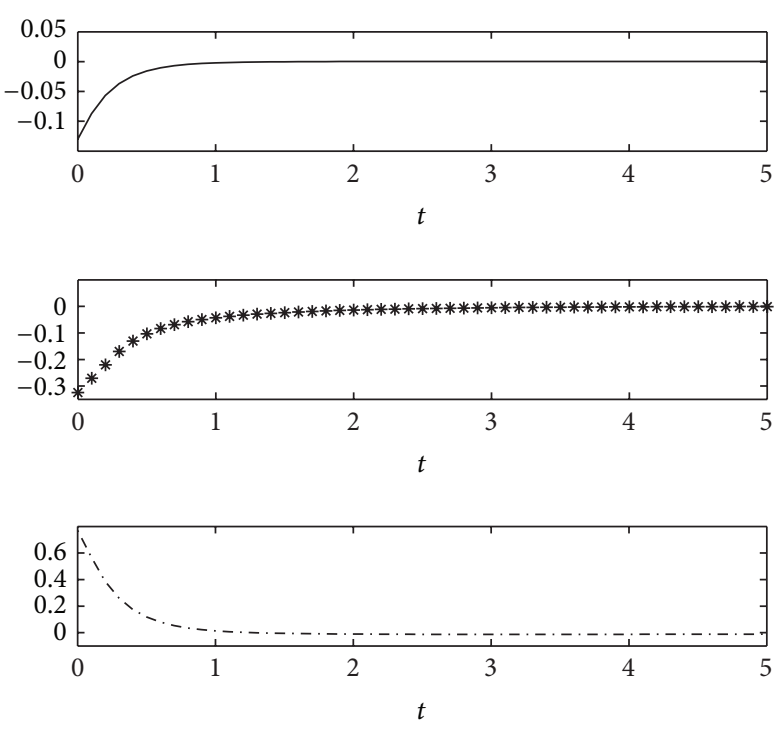

FIGURE 6: The trajectories of $e_{1}$ (solid line), $e_{2}$ (star line), and $e_{3}$ (dash-dotted line).

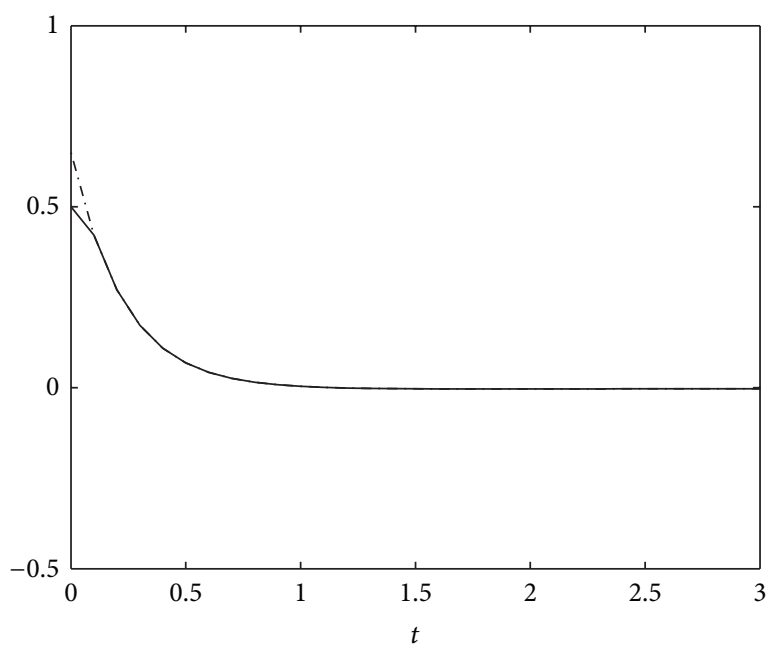

FIgURE 7: The trajectories of $v_{1}$ (solid line) and $u_{1}$ (dash-dotted line).

\section{Simulation Results 2}

In this section, the initial values are chosen as $x_{1}(0)=0.82$, $y_{1}(0)=0.29, z_{1}(0)=0.48, x_{2}(0)=0.69, y_{2}(0)=-0.03$, and $z_{2}(0)=1.25$, and the other initial values are chosen as zeros. The saturation values are $u_{1 M}=0.5, u_{2 M}=0.5$, and $u_{3 M}=2$. The design parameters in controllers are $\bar{l}_{1}=5, \bar{l}_{2}=4$, and $\bar{l}_{3}=4$. The simulation results are shown in Figures $6,7,8$, and 9.

\section{Conclusions}

For a class of known and unknown parameters for energy resource demand-supply system with input constraints, the chaos synchronization problems have been discussed using two different control methods, respectively. The main features 


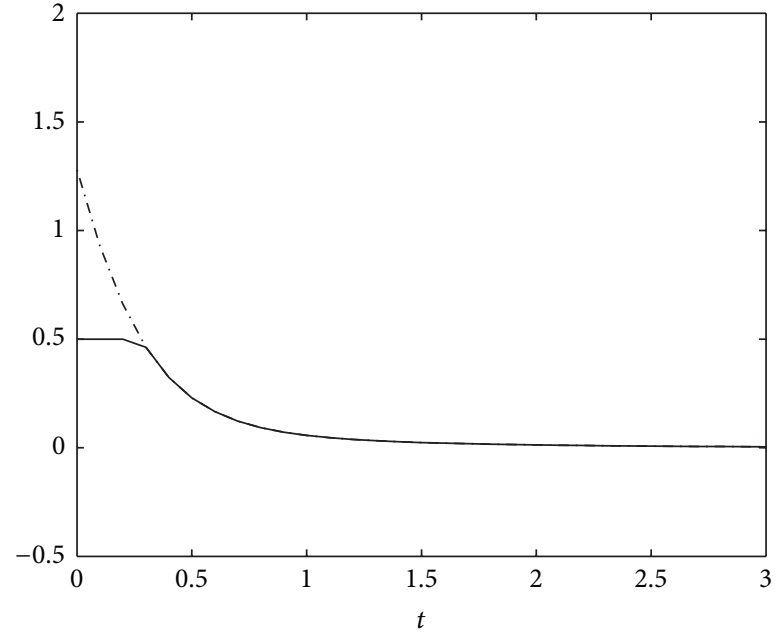

FIGURE 8: The trajectories of $v_{2}$ (solid line) and $u_{2}$ (dash-dotted line).

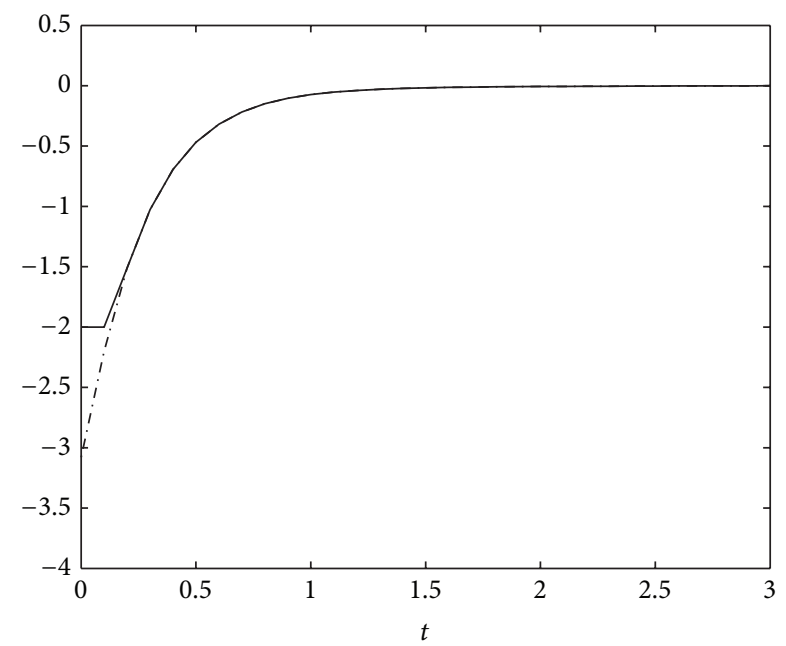

FIGURE 9: The trajectories of $v_{3}$ (solid line) and $u_{3}$ (dash-dotted line).

of the proposed algorithm are that (i) the problems of the input constraint have been solved by employing a new auxiliary system; (ii) the stability of the energy resource demandsupply system has been guaranteed based on Lyapunov theory. The results all have demonstrated the validity and feasibility of the proposed approaches.

\section{Acknowledgment}

This work was supported by the Foundation of Liaoning Educational Committee (no. L2012225).

\section{References}

[1] M. Sun, L. Tian, Y. Fu, and W. Qian, "Dynamics and adaptive synchronization of the energy resource system," Chaos, Solitons and Fractals, vol. 31, no. 4, pp. 879-888, 2007.
[2] M. Sun, L. Tian, and Y. Fu, "An energy resources demand-supply system and its dynamical analysis," Chaos, Solitons and Fractals, vol. 32, no. 1, pp. 168-180, 2007.

[3] M. Sun, Q. Jia, and L. Tian, "A new four-dimensional energy resources system and its linear feedback control," Chaos, Solitons and Fractals, vol. 39, no. 1, pp. 101-108, 2009.

[4] C. Huang, K. Cheng, and J. Yan, "Robust chaos synchronization of four-dimensional energy resource systems subject to unmatched uncertainties," Communications in Nonlinear Science and Numerical Simulation, vol. 14, no. 6, pp. 2784-2792, 2009.

[5] M. Sun, L. Tian, and J. Xu, "Time-delayed feedback control of the energy resource chaotic system," International Journal of Nonlinear Science, vol. 1, no. 3, pp. 172-177, 2006.

[6] X. Li, W. Xu, and R. Li, "Chaos synchronization of the energy resource system," Chaos, Solitons and Fractals, vol. 40, no. 2, pp. 642-652, 2009.

[7] A. Boulkroune, M. M'Saad, and M. Farza, "Adaptive fuzzy controller for multivariable nonlinear state time-varying delay systems subject to input nonlinearities," Fuzzy Sets and Systems, vol. 164, no. 1, pp. 45-65, 2011.

[8] C. Wen, J. Zhou, Z. Liu, and H. Su, "Robust adaptive control of uncertain nonlinear systems in the presence of input saturation and external disturbance," Institute of Electrical and Electronics Engineers, vol. 56, no. 7, pp. 1672-1678, 2011.

[9] C.-S. Ting, "A robust fuzzy control approach to stabilization of nonlinear time-delay systems with saturating inputs," International Journal of Fuzzy Systems, vol. 10, no. 1, pp. 50-60, 2008.

[10] S. C. Tong and Y. M. Li, "Adaptive fuzzy output feedback control of MIMO nonlinear systems with unknown dead-zone input," IEEE Transactions on Fuzzy Systems, vol. 21, no. 1, pp. 134-146, 2013.

[11] S. Tong and Y. Li, "Adaptive fuzzy output feedback tracking backstepping control of strict-feedback nonlinear systems with unknown dead zones," IEEE Transactions on Fuzzy Systems, vol. 20, no. 1, pp. 168-180, 2012.

[12] T. Li, J. Yu, and Z. Wang, "Delay-range-dependent synchronization criterion for Lur'e cprime e systems with delay feedback control," Communications in Nonlinear Science and Numerical Simulation, vol. 14, no. 5, pp. 1796-1803, 2009.

[13] T. Li and W. X. Zheng, "New stability criterion for fixed-point state-space digital filters with generalized overflow arithmetic," IEEE Transactions on Circuits and Systems B, vol. 59, no. 7, pp. 443-447, 2012.

[14] Y. Li, S. Tong, and T. Li, "Adaptive fuzzy output feedback control of uncertain nonlinear systems with unknown backlash-like hysteresis," Information Sciences, vol. 198, pp. 130-146, 2012.

[15] Y. M. Li, T. S. Li, and X. J. Jing, "Indirect adaptive fuzzy control for input and output constrained nonlinear systems using a barrier Lyapunov function," International Journal of Adaptive Control and Signal Processing, 2013.

[16] J. H. Pérez-Cruz, E. Ruiz-Velázquez, J. J. Rubio, and C. A. de Alba-Padilla, "Robust adaptive neurocontrol of SISO nonlinear systems preceded by unknown deadzone," Mathematical Problems in Engineering, vol. 2013, Article ID 342739, 23 pages, 2012.

[17] H. W. Zhu, G. Z. Qin, Y. X. Yan, Z. C. Jiang, and Z. S. Duan, "Delayed antiwindup control using a decoupling structure," Mathematical Problems in Engineering, vol. 2013, Article ID 248153, 7 pages, 2013. 
[18] T. Wang, S. Tong, and Y. Li, "Robust adaptive fuzzy control for nonlinear system with dynamic uncertainties based on backstepping," International Journal of Innovative Computing, Information and Control, vol. 5, no. 9, pp. 2675-2688, 2009.

[19] S. Tong and Y. Li, "Observer-based fuzzy adaptive control for strict-feedback nonlinear systems," Fuzzy Sets and Systems, vol. 160, no. 12, pp. 1749-1764, 2009. 


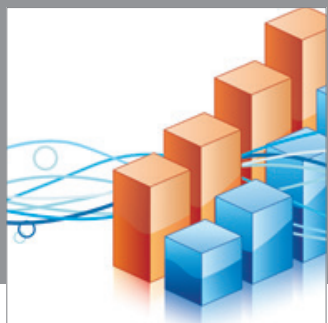

Advances in

Operations Research

mansans

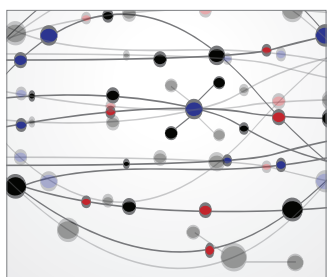

The Scientific World Journal
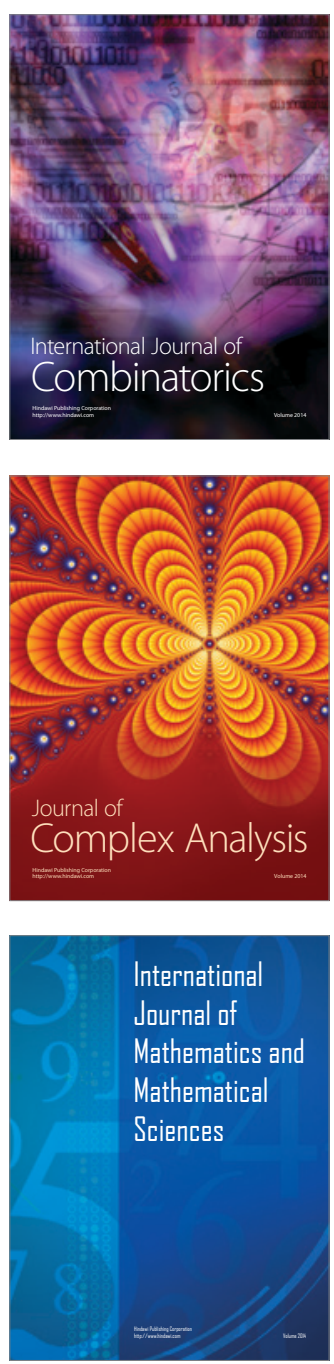
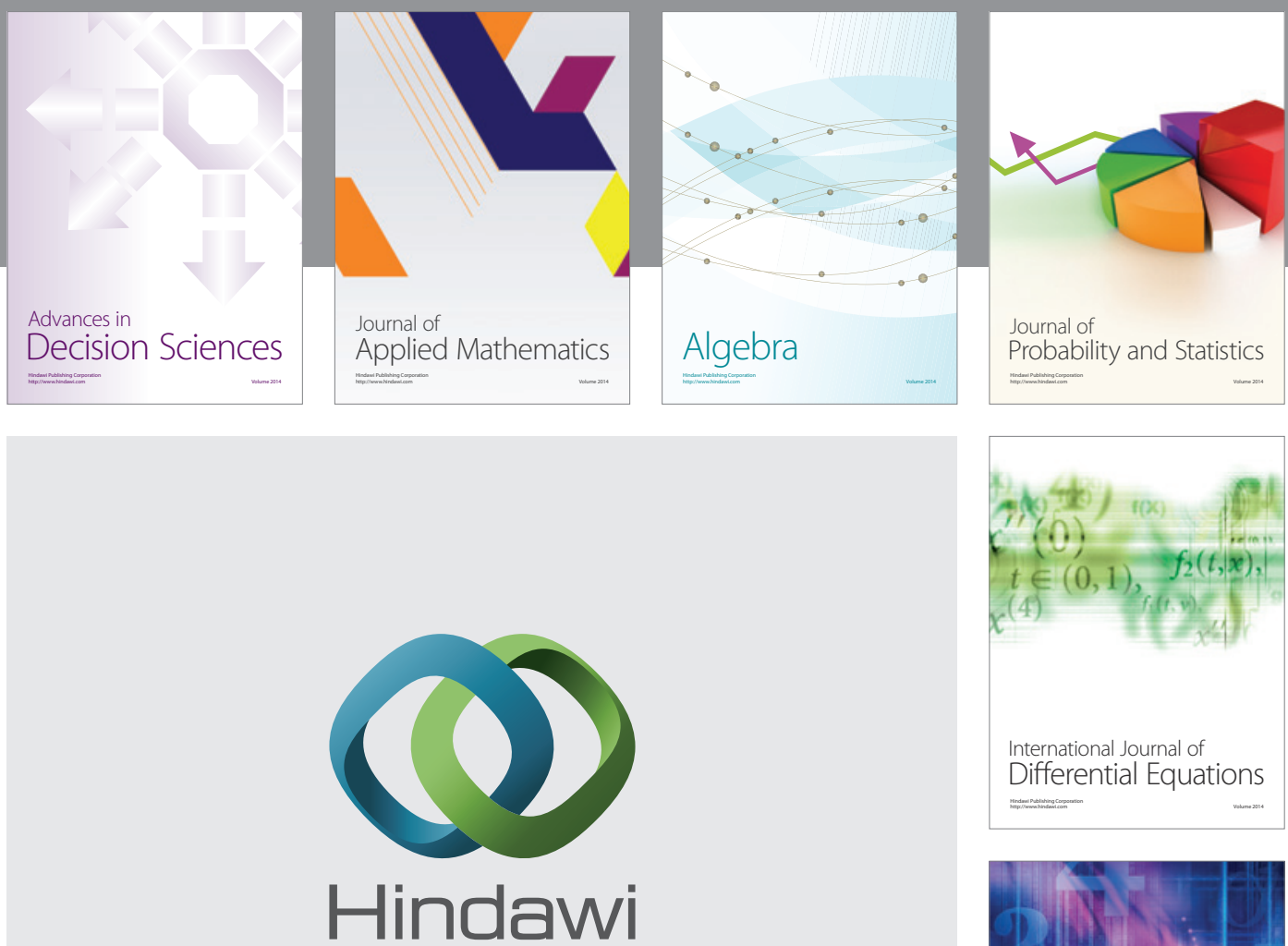

Submit your manuscripts at http://www.hindawi.com
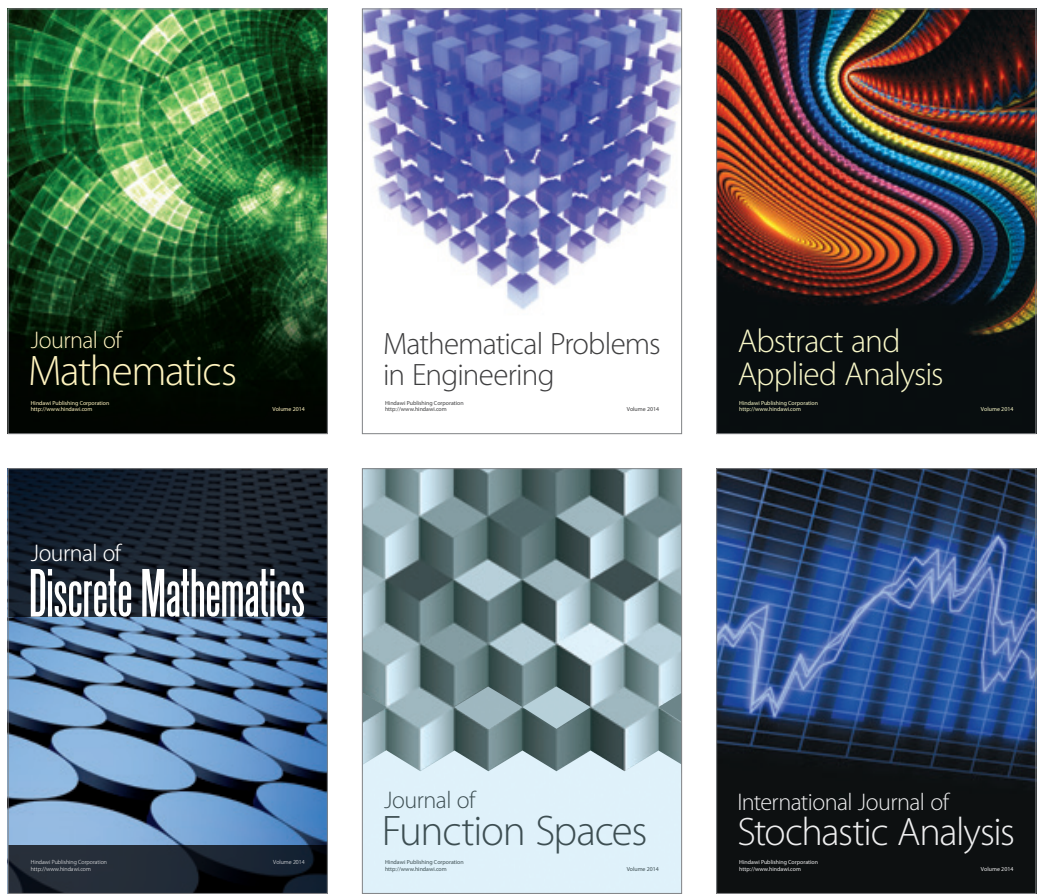

Journal of

Function Spaces

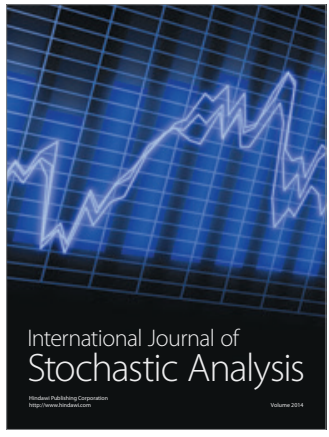

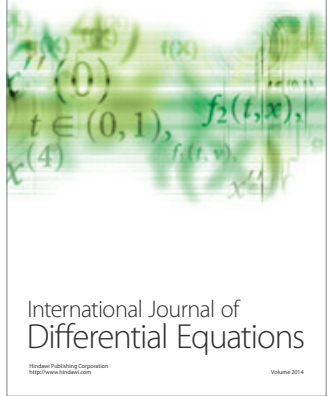
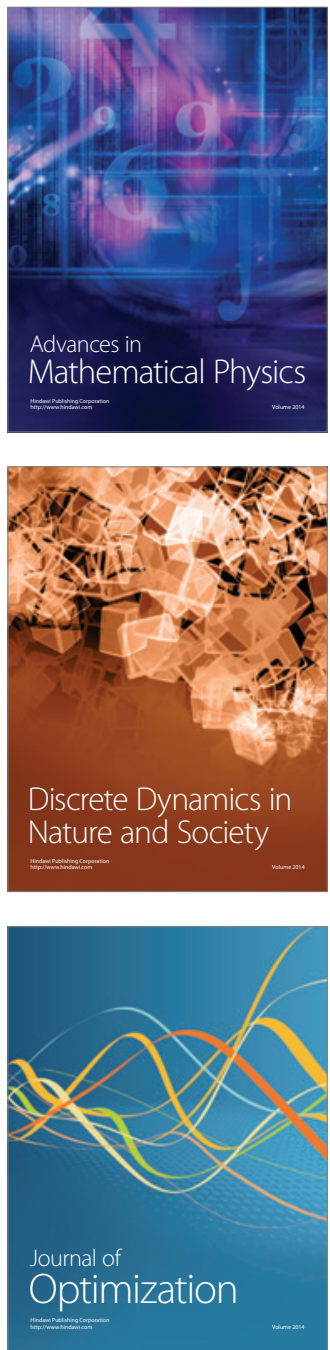